\title{
Trabajo y plataformas de servicios en la economía colaborativa: una oportunidad para emprendedores
}

\author{
Jacques Bulchand-Gidumal ${ }^{1}$, Santiago Melián-González \\ Universidad de Las Palmas de Gran Canaria (ULPGC), España
}

doi: $10.20420 /$ eni.2019.278

\begin{abstract}
Resumen
Economía bajo demanda y economía del trabajo ocasional son dos de los términos que se han usado para referirse a la faceta laboral de la economía colaborativa. Algunas de las plataformas más relevantes de esta economía (ej., TaskRabbit y Upwork) son ya bastante populares. El auge de esta nueva forma de trabajar ha hecho que se hable de mercado laboral digital para diferenciarlo del tradicional. Este estudio recopila las características atribuidas al trabajo que se intercambia en el mercado laboral digital y las analiza utilizando datos de 465 trabajadores. Nuestros resultados confirman y refinan algunas creencias existentes en la literatura.
\end{abstract}

Palabras clave: economía bajo demanda conomía colaborativa, trabajo no estándar, mercado laboral digital.

Clasificación JEL: J01, J21

Fuente de referencia: Melián-González, S., \& Bulchand-Gidumal, J. (n.d.). What type of labor lies behind the on-demand economy? New research based on workers’ data. Journal of Management \& Organization, 1-17. doi:10.1017/jmo.2018.53

\section{Introducción}

Las grandes innovaciones tecnológicas siempre han tenido consecuencias sobre el mercado laboral, destruyendo algunas ocupaciones, servicios y tareas y creando otras nuevas. En el caso de Internet, una de las consecuencias que ha producido es la llamada economía colaborativa, que se define como aquella en la que "[...] se produce la venta, intercambio o cesión de productos o servicios, principalmente por parte de individuos, mediante plataformas tecnológicas en línea que permiten la conexión y gestión de la relación entre los proveedores y los consumidores, usando los proveedores medios propios, disponiendo de autonomía para organizarse y sin alcanzar el tamaño de una pyme [...]" (Bulchand-Gidumal y Melián-González, 2018:29).

Mientras que algunas de las plataformas más populares de esta economía colaborativa están orientadas al intercambio y alquiler de bienes (por ejemplo, Airbnb, Etsy o HomeExchange), en muchas otras se ofrecen y demandan servicios. Estos son proporcionados por personas. A partir de estas plataformas, se ha propuesto que está emergiendo un nuevo tipo de trabajo, claramente temporal y bajo demanda, que se han encuadrado dentro de la denominada economía bajo demanda o economía del trabajo ocasional. Dado que las personas pueden ofrecer un amplio abanico de tareas, con diferentes condiciones, en este contexto digital se ha propuesto el término mercado laboral digital (MLD) (Codagnone, Abadie y Biagi, 2016) para definir este fenómeno que, a su vez, se une a las opciones de trabajo no estándar2 ya existentes. Estos habitualmente se han calificado como trabajos secundarios, asociados a características negativas tales como salarios bajos, precariedad,

\footnotetext{
${ }^{1}$ Autor de correspondencia: jacques.bulchand@ulpgc.es

${ }^{2}$ Se define trabajo no estándar como todo aquel que no es permanente y a tiempo completo (Horemans, 2016). Se incluyen en este grupo los contratos temporales, los contratos basados en agencias de trabajo temporal, los procesos de externalización y los autónomos independientes, entre otros.
} 
inestabilidad y pocas oportunidades de promoción (Doeringer y Piore, 1971).

El MLD se sustenta en un modelo novedoso basado en plataformas digitales en las que los clientes pueden solicitar una gran variedad de servicios bajo demanda. Estos se basan en el trabajo y van desde sencillas tareas físicas e intelectuales hasta proyectos que requieren cualificación universitaria. Las expectativas son de un crecimiento significativo en los próximos años. Precisamente su novedad hace que los estudios existentes hasta la fecha sean escasos y basados en evidencias poco sólidas. Aun así, los resultados existentes parecen confirmar las características negativas asociadas a esta nueva forma de trabajar.

Por ello, el objetivo de este estudio es analizar las características del trabajo en el MLD usando una muestra amplia de datos de los trabajadores de las plataformas. Intentando salvar las dificultades de los estudios existentes, nos hemos basado en datos de trabajadores de dos plataformas importantes y diferentes entre sí: TaskRabbit y Trip4Real (actualmente, parte de Airbnb Experiences).

\section{Mercado laboral digital}

En general, se considera que el mercado laboral digital acoge a una forma de trabajar que comparte características con las formas de trabajo atípicas ya conocidas. Este mercado laboral digital puede ser clasificado en base a dos parámetros. Por un lado, dependiendo del lugar en que se desempeña el trabajo, tenemos el mercado virtual y el mercado presencial. Por otro, dependiendo del nivel de cualificación necesario para desarrollar las tareas, tenemos el mercado de baja cualificación y el mercado de alta cualificación.

Por tanto, aparecen cuatro MLD independientes (Codagnone y otros, 2016): mercado virtual que exige un nivel de cualificación bajo a medio (por ejemplo, Amazon Mechanical Turk); mercado presencial que exige también un nivel de cualificación bajo a medio (TaskRabbit); mercado virtual que exige un nivel de cualificación medio a alto (Upwork); y mercado presencial que exigen un nivel de cualificación medio a alto (Takelessons).

El trabajo en los anteriores segmentos del MLD puede incluir condiciones laborales positivas y negativas, si bien las características negativas parecen prevalecer. Entre las positivas, cabe mencionar la flexibilidad y autonomía del trabajador (cuestión que queda en entredicho cuando las plataformas aplican un control excesivo sobre el trabajador).

Entre las negativas se pueden citar el bajo nivel de protección social, la escasa duración de las tareas, la incertidumbre, el alto nivel de competición entre los trabajadores debido al exceso de oferta, el escaso poder negociador de los trabajadores, las largas jornadas laborales, la subordinación a la experiencia del cliente y la posibilidad de que las ganancias no sean suficientes para alcanzar unos ingresos dignos.

Los estudios disponibles hasta el momento basados en datos de los trabajadores (Hall y Krueger, 2015; De Groen y otros, 2016; Maselli y Fabo, 2015; Berg, 2016) confirman, en general, las citadas características negativas. No obstante, todos ellos tienen carencias metodológicas significativas.

\section{Metodología}

En enero de 2016 se descargaron datos de dos plataformas: TaskRabbit (mercado presencial de baja a media cualificación) y Trip4Real (que actualmente es parte de Airbnb Experiences y se asignaría al mercado presencial de media a alta cualificación). Se seleccionaron datos de trabajadores con 15 o más valoraciones por parte de los clientes. En el caso de TaskRabbit, se descargaron datos de 290 trabajadores de 10 
grandes ciudades de Estados Unidos. En el caso de Trip4Real, se descargaron datos de 175 trabajadores de 16 destinos turísticos.

\section{Resultados}

En ambas plataformas la mayor parte de los trabajadores se unieron a las mismas en 2014 y 2015. En el caso de TaskRabbit, la muestra de trabajadores seleccionada ha trabajado de media unas 480 horas, distribuidas en unas 240 tareas, existiendo algunos trabajadores que han desarrollado hasta casi 1.800 tareas. Se asume una duración media de las tareas de unas dos horas. Anualmente, la media es de unas 295 horas trabajadas, con un máximo anual de 1.240 horas. En el caso de Trip4Real, los números son muy inferiores.

En cuanto al ratio de tareas que son valoradas por los clientes, este se sitúa en 0,61. Es decir, el $61 \%$ de las tareas que se realizan son valoradas por los clientes.

Analizando el tipo de tareas realizadas, los datos descargados de TaskRabbit muestran hasta 44 tipos de tareas distintas. 4 de ellas son muy frecuentes y se llevan a cabo por más de la mitad de los trabajadores: entregas, montaje de muebles, mudanzas y movimiento de muebles. En cambio, son poco frecuentes tareas como diseño web, diseño gráfico, cuidado de personas mayores o contabilidad. 8 tareas representan el $82,3 \%$ de todo el trabajo que fue valorado: las cuatro citadas arriba más pequeños arreglos domésticos, limpieza, montajes varios y tareas de jardinería.

En el caso de Trip4Real, la tarea más común son las rutas turísticas (más del 60\% del total), siendo otras actividades mucho menos habituales (rutas gastronómicas, deporte y aventuras, vida nocturna, etc.). De media, las tareas desarrolladas tienen una duración de 3,6 horas.

Analizando los ingresos generados, encontramos que el trabajador que realizó más tareas a lo largo de un año en TaskRabbit habría obtenido un ingreso bruto anual cercano a los 63 mil dólares. El trabajador situado en la mediana tendría unos ingresos brutos anuales de 15 mil dólares. Hay que tener en cuenta que las plataformas se quedan con entre un 15 y un 30\% de estas cifras y que, además, los trabajadores tienen que deducir de las mismas sus gastos: seguridad social, desplazamientos, equipamiento, material de trabajo, etc.

\section{Conclusiones}

Las plataformas digitales facilitan la conexión entre la oferta y la demanda. En el caso de las relaciones de trabajo, permiten que los trabajadores ofrezcan servicios que satisfagan todo tipo de demandas de los clientes y que estos encuentren fácilmente trabajadores capaces de desarrollar las tareas que necesitan. La presencia de valoraciones del trabajo realizado es una novedad que, hasta ahora, no había existido en el mercado laboral tradicional.

Aunque se ha explicado que el trabajo en el MLD puede considerarse una forma adicional de trabajo no estándar, una cuestión nueva es que los trabajadores en el MLD suelen realizar conjuntos de tareas poco relacionadas entre sí. Hasta ahora, el trabajo no estándar se ha caracterizado por desarrollar agrupaciones de tareas asociadas a una ocupación. Se han contratado directamente o a través de empresas de trabajo temporal (por ejemplo, en el ámbito de la cocina, de la jardinería, o de la limpieza). En cambio, en el caso de TaskRabbit encontramos trabajadores que desempeñan tareas propias de diferentes ocupaciones.

Algunas de las características negativas que se habían atribuido en la literatura al MLD se han confirmado, pero con matizaciones. En primer lugar, el MLD no es solo de trabajo de baja cualificación; en el mismo encontramos plataformas que incluyen tareas que requieren un amplio rango de cualificaciones e, incluso, 
plataformas que solo intermedian en trabajo de alta cualificación.

Sí se ha confirmado que la carga de trabajo no es comparable a la de una relación laboral tradicional a tiempo completo. Trabajar en el MLD parece más bien una ocupación a tiempo parcial. Si esta situación se da por decisión de los trabajadores o por una baja demanda es algo que no hemos podido confirmar. Aun así, los datos encontrados en el presente estudio en cuanto a número de tareas realizadas son muy superiores a las cifras encontradas en investigaciones previas (De Groen y otros, 2016; Maselli y Fabo, 2015). También se ha confirmado que la mayoría de las tareas que se solicitan son de corta duración.

En el mismo sentido, las cifras de ingresos encontradas también son significativamente más elevadas que las citadas en la literatura (De Groen y otros, 2016; Maselli y Fabo, 2015). Aun así, no equivalen, en la mayoría de los casos, a las de un trabajo a tiempo completo. Parece claro que los trabajadores del MLD deberán trabajar en varias plataformas simultáneamente, o bien complementar sus ingresos con otras tareas realizadas al margen de este mercado.

No obstante, es necesario recalcar que, aunque las cifras de ingresos totales son bajas, las cifras de ingreso por hora no lo son. Hemos encontrado tareas retribuidas a importes cercanos a los 40 dólares por hora, que es muy superior a los 20 o 30 dólares por hora de salario mínimo por esas mismas tareas en Estados Unidos.

En cuanto al nivel de competencia existente, nuestros datos demuestran que es cierto, que un gran número de trabajadores realizan tareas muy similares en zonas geográficas cercanas, compitiendo, por tanto, por los mismos clientes. $\mathrm{Y}$ se verifica la existencia de un efecto superestrella: la mayor parte de los encargos lo asume un pequeño porcentaje de los trabajadores disponibles. Dicho de otra forma, la oferta supera muy ampliamente a la demanda. El hecho de contar con ninguna o pocas valoraciones puede estar dificultando el acceso a los encargos por parte de los más novatos en el MLD.

En este sentido, también hemos confirmado un elevado nivel de subordinación a la experiencia del cliente. Tener una mala valoración puede afectar significativamente a las posibilidades futuras de empleo.

En resumen, nuestra investigación demuestra que el MLD tiene el potencial de ser una forma de ganarse la vida, visto que el ingreso por hora es elevado. Pero la realidad es más compleja. La mayoría de las tareas son de corta duración, el nivel de competencia es muy elevado, se da el efecto superestrella $y$ hay una elevada subordinación a la experiencia del cliente.

Por ello, para que un trabajador pueda vivir del MLD deberá desempeñar tareas en varias plataformas o bien deberá ser capaz de encontrar una estrategia de diferenciación que permita que su oferta destaque en las mismas. Dicho de otra forma, probablemente, en la actualidad, el MLD sea más apropiado como fuente de ingresos complementarios.

\section{Lecciones y oportunidades para emprendedores}

Las principales conclusiones del presente estudio, presentadas en el apartado anterior, permiten alumbrar una serie de cuestiones de interés para los emprendedores.

En primer lugar, ha quedado demostrado y claro que el MLD está creciendo y crecerá significativamente en los próximos años. La experiencia pasada y los paralelismos con otros ámbitos digitales permiten afirmar que estamos ante un sector que aún se encuentra en pleno proceso de desarrollo. Por ello, las oportunidades de creación de nuevos modelos de negocio alrededor de las plataformas laborales digitales son amplias. Tanto porque hay sectores $\mathrm{y}$ 
ámbitos geográficos por servir, como porque empiezan a aparecer nuevos paradigmas que tratan de corregir los defectos actuales del MLD, con modelos en los que el trabajador tiene una mayor cuota de participación en las decisiones y mejor cobertura social. También creemos necesario citar la existencia de nuevas tecnologías que suponen una oportunidad de mejora en este ámbito, tanto para las plataformas actuales como en el desarrollo de nuevas plataformas. Entre otras muchas, mencionamos el blockchain, la ciencia de datos (big data) y la inteligencia artificial.

En segundo lugar, el MLD se muestra como una excelente opción para adquirir experiencia y lograr ingresos complementarios. Muchos emprendedores que están en los estadios iniciales de desarrollo de sus modelos de negocio disponen de una oportunidad de aprender y de obtener algunos ingresos ocasionales, utilizando sus competencias (por ejemplo, programación o diseño gráfico). Estos ingresos probablemente les puedan ayudar en estas etapas iniciales y servir de sustento al proceso de desarrollo del negocio principal.

\section{Referencias}

Berg, J. (2016). Income security in the on-demand economy: Findings and policy lessons from a survey of crowdworkers, International Labour Organization. Geneva. Disponible en http://www.ilo.org/wcmsp5/groups/public/--ed_protect/---protrav/---

travail/documents/publication/wcms_479693.pdf
Bulchand-Gidumal, J. y Melián-González, S. (2018). La revolución de la economía colaborativa. Editorial LID, Madrid.

Codagnone, C., Abadie, F. y Biagi F. (2016). The Future of Work in the 'Sharing Economy'. Market Efficiency and Equitable Opportunities or Unfair Precarisation? Institute for Prospective Technological Studies, JRC Science for Policy Report EUR 27913 EN. Disponible en https://papers.ssrn.com/sol3/papers.cfm?abstract_ id $=2784774$.

De Groen, W.P., Maselli, I. y Fabo, B. (2016). The Digital Market for Local Services: A one night stand for workers? CEPS Special Report No. 133. Disponible

en https://papers.ssrn.com/sol3/papers.cfm?abstract_ id $=2766220$.

Doeringer, P.P. y Piore, M.J.M. (1971). Internal labor markets and manpower analysis. New York: D.C. Heath and Company.

Hall, J.V. y Krueger, A.B. (2015). An analysis of the labor market for Uber's driver-partners in the United States.

Disponible en https://assets.documentcloud.org/documents/1507 970/uberstudy.pdf.

Horemans, J. (2016). Polarisation of non-standard employment in Europe: Exploring a missing piece of the inequality puzzle. Social Indicators Research, 125(1), 171-189.

Maselli, I. y Fabo, B. (2015). Digital workers by design? An example from the on-demand economy. CEPS Working Document No. 414/October 2015. Disponible en http://aei.pitt.edu/68757/. 